\title{
A Quality Assessment of a Collaborative Model of a Pediatric Antimicrobial Stewardship Program
}

\author{
Phuong-Tan Nguyen-Ha, PharmD, ${ }^{a}$ Denise Howrie, PharmD, ${ }^{a}$ Kelli Crowley, PharmD, ${ }^{a}$ Carol G. Vetterly, PharmD, ${ }^{a}$ \\ William McGhee, PharmD, ${ }^{a}$ Donald Berry, RPh, ${ }^{a}$ Elizabeth Ferguson, PharmD, ${ }^{a}$ Emily Polischuk, Pharm D, ${ }^{a}$ \\ Maria Mori Brooks, PhD, ${ }^{b}$ Jeffrey Goff, RPh, MS, ${ }^{a}$ Terri Stillwell, MD, MPH, ${ }^{\mathrm{c}}$ Toni Darville, MD, ${ }^{\mathrm{d}}$ Ann E. \\ Thompson, MD, ${ }^{e}$ James E. Levin, MD, PhD, ${ }^{f, \dagger}$ Marian G. Michaels, MD, MPH, ${ }^{f, g}$ Michael Green, MD, MPH,
}

BACKGround: Infectious Diseases Society of America guidelines recommend that key antimicrobial stewardship program (ASP) personnel include an infectious disease (ID) physician leader and dedicated ID-trained clinical pharmacist. Limited resources prompted development of an alternative model by using ID physicians and service-based clinical pharmacists at a pediatric hospital. The aim of this study was to analyze the effectiveness and impact of this alternative ASP model.

METHODS: The collaborative ASP model incorporated key strategies of education, antimicrobial restriction, day 3 audits, and practice guidelines. High-use and/or high-cost antimicrobial agents were chosen with audits targeting vancomycin, caspofungin, and meropenem. The electronic medical record was used to identify patients requiring day 3 audits and to communicate ASP recommendations. Segmented regression analyses were used to analyze quarterly antimicrobial agent prescription data for the institution and selected services over time.

RESULTS: Initiation of ASP and day 3 auditing was associated with blunting of a preexisting increasing trend for caspofungin drug starts and use and a significant downward trend for vancomycin drug starts (relative change $-12 \%)$ and use $(-25 \%)$, with the largest reduction in critical care areas. Although meropenem use was already low due to preexisting requirements for preauthorization, a decline in drug use $(-31 \%, P=.021)$ and a nonsignificant decline in drug starts $(-21 \%, P=.067)$ were noted. A 3-month review of acceptance of ASP recommendations found rates of $90 \%, 93 \%$, and $100 \%$ for vancomycin, caspofungin, and meropenem, respectively.

CONCLUSIONS: This nontraditional ASP model significantly reduced targeted drug usage demonstrating acceptance of integration of service-based clinical pharmacists and ID consultants.

In 2007, the Infectious Diseases Society of America (IDSA) and the Society for Healthcare Epidemiology of America jointly published the first guidelines for formation of antimicrobial stewardship programs (ASPs) intended to optimize patient care outcomes while minimizing antimicrobial resistance and drug toxicity. ${ }^{1}$ ASPs have proven beneficial in reducing the frequency and duration of target drug prescribing, occurrence of antiinfective-related infectious complications (eg, Clostridium difficile-associated disease) and health care costs with

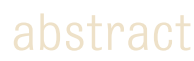

abstract

\begin{abstract}
a Department of Pharmacy, Children's Hospital of Pittsburgh, Pittsburgh, Pennsylvania; ${ }^{b}$ Division of Biostatistics, Graduate School of Public Health, University of Pittsburgh, Pittsburgh, Pennsylvania; ' Department of Pediatrics, University of Michigan, Ann Arbor, Michigan; ${ }^{d}$ Department of Pediatrics, University of North Carolina School of Medicine, Chapel Hill, North Carolina; and Departments of ${ }^{e}$ Critical Care Medicine, fSurgery, and gPediatrics, University of Pittsburgh School of Medicine, Pittsburgh, Pennsylvania

\section{†Deceased.}

Dr Nguyen-Ha conceptualized and designed the study, carried out portions of the initial analysis, drafted the initial manuscript, actively participated in the development of the antimicrobial stewardship program (ASP) and its implementation, and approved the final manuscript as submitted; Drs Howrie, Crowley, Vetterly, McGhee, Ferguson, and Polischuk actively participated in the initial design of the study and data analyses, provided serial comments and recommendations and actively reviewed and edited the manuscript from the point of seeing the initial draft until its completion, and approved the final manuscript as submitted; Mr Goff and Mr Berry participated in the initial design of the ASP and in its ongoing assessment, provided serial comments and recommendations and actively reviewed and edited the manuscript from the point of seeing the initial draft until its completion, and approved the final manuscript as submitted; Dr Stillwell helped to conceptualize and implement the ASP as well as worked to develop the mechanisms of analyses using the electronic medical record, provided serial comments and recommendations and actively reviewed and edited the manuscript from the point of seeing the initial draft until its completion, and approved

\footnotetext{
To cite: Nguyen-Ha P, Howrie D, Crowley K, et al. A Quality Assessment of a Collaborative Model of a Pediatric Antimicrobial Stewardship Program. Pediatrics. 2016;137(5):e20150316
} 
recent attention focusing on the role of ASP in quality improvement and patient safety. ${ }^{2}$ ASP programs in pediatric institutions have also favorably affected antimicrobial usage $^{3-7}$ and recommendations for ASP development specific to pediatrics have been authored, ${ }^{8,9}$ citing the importance of participation of an infectious disease (ID) physician and a trained clinical pharmacist. ${ }^{3,4}$ However, limited availability of pediatric ID trained pharmacists and limited institutional resources may require modification of this classic ASP model.1,8-10

In 2008, the Children's Hospital of Pittsburgh of University of Pittsburgh Medical Center (UPMC) established an innovative collaborative model of shared ASP responsibilities by ID physicians and service-based clinical pharmacists under the direction of an ID physician leader and advisory committee. The program was developed from its onset to leverage the hospital's electronic medical record (EMR) and data warehouse to facilitate ongoing evaluations of the ASP to inform potential additional interventions.

The aim of this study was to analyze the effectiveness of this alternative ASP model in improving the appropriateness of starts and overall use for 3 targeted antimicrobial agents.

\section{METHODS}

\section{Setting}

The Children's Hospital of Pittsburgh of UPMC is a free-standing hospital with 315 beds, including 79 total ICU beds (36 PICU, 12 cardiac ICU, and 31 NICU). The hospital provides inpatient care for a wide range of clinical conditions from acute medical problems to the management of complex chronic diseases, including children with cancer and those undergoing solid organ and stem cell transplantation. Additionally, there are general surgery, neonatal surgery, cardiothoracic surgery, and orthopedic and trauma populations treated.

\section{ASP Program Development:}

In 2008, an ASP advisory committee representing clinical resource management, pharmacy services, ID, infection prevention, and medical informatics devised a unique model by using physician faculty and fellows and a team of service-based clinical pharmacists responsible for patient populations including critical care, oncology/ bone marrow transplantation, solid organ transplantation, and medical pediatrics. The physician director of the new ASP was provided 10\% full-time equivalent (FTE) support but no other additional salary support was provided for the ASP. Preexisting antimicrobial restriction and preauthorization practices were continued. New activities included development of practice guidelines and implementation of prospective day 3 audits of targeted agents with education and feedback. Caspofungin, vancomycin, and meropenem were selected for auditing based on an assessment of usage patterns, cost impact, and/or potential for acquired drug resistance. ASP members collaborated with key physician services to develop guidelines emphasizing recommendations for initial as well as continued use at 72 hours of treatment of these antimicrobial agents. Guideline development for caspofungin began in January 2009 with implementation of day 3 auditing the same month. The efforts for vancomycin began in February 2009 with implementation of day 3 auditing in April 2010.

Finally, efforts for meropenem began in August 2010 with initiation of day 3 auditing in October 2010.

\section{Data and Measures}

Data collection was facilitated through use of the EMR (Cerner,
Kansas City, M0); daily data warehouse reports were generated identifying patients requiring a day 3 audit. A customized ASP electronic note (eNote) template was constructed, permitting documentation of ASP recommendations in the EMR and also data collection for ASP program analysis. eNotes provided a recommendation to discontinue, continue, switch to an alternate antimicrobial, and/or consult ID with the option to include duration of treatment. Recommendations for dose adjustments were made by pharmacists in separate eNotes. Audits were completed by clinical pharmacists during the week and by ID physicians/fellows on weekends and holidays. Clinical status and potential indications for antimicrobial use were discussed as part of daily work rounds for those services with designated clinical pharmacists. An on-call ASP pharmacist performed day 3 audits for all other patients. ASP recommendations were discussed with ID physicians as needed and communicated to the primary service both verbally and via eNote.

The data warehouse was further leveraged to evaluate ASP impact through the generation of quarterly reports assessing key endpoints including but not limited to drug starts (patients receiving drug per quarter/1000 patients per quarter) and drug use (days of therapy per quarter/1000 patientdays per quarter). Preintervention antimicrobial use data were collected from the first quarter of 2008 for caspofungin and vancomycin and from the first quarter of 2009 for meropenem through the initiation of day 3 auditing for each targeted antimicrobial. Postintervention data were collected from the implementation of day 3 auditing (first quarter of 2009 for caspofungin, second quarter of 2010 for vancomycin, and fourth quarter 
of 2010 for meropenem) through the third quarter of 2013 for all 3 antimicrobial agents. Hospitalwide and service-specific results for the targeted antimicrobial agents and other agents of interest (eg, piperacillin/tazobactam, ciprofloxacin) were reviewed on a quarterly basis to assess progress toward optimizing antimicrobial use. Unexpected increases in use of antimicrobial agents were discussed and educational interventions were implemented where appropriate with subsequent follow-up to determine the effectiveness of these additional efforts.

To assess acceptance of ASP recommendations, a chart review was conducted on all patients who had an ASP eNote written from October through December 2011. Acceptance of ASP recommendation was defined as following the recommendation within 24 hours of completion of eNote. Compliance was calculated as the percentage of ASP recommendations accepted versus number of eNotes written over the 3-month period for each antimicrobial.

\section{Statistical Methods}

Rates of drug starts and drug use were computed for each of the targeted antimicrobial agents on a quarterly basis. For each antimicrobial, 2-sample $t$ tests were conducted to compare quarterly drug start and drug use rates before and after the ASP intervention. Segmented regression analyses ${ }^{11,12}$ were used to analyze the "interrupted time series" of quarterly antimicrobial prescriptions. Full segmented regression models included a preintervention intercept, a preintervention slope, a change in level after ASP (ie, a jump), and a change in slope post-ASP. Backward stepwise regression methods were used to create a parsimonious segmented regression models by eliminating parameters with $P>$
.10. Data for individual services were examined as exploratory data analyses. Statistical analysis was carried out by using SAS 9.2 (SAS Institute, Inc, Cary, NC) and $P$ $<.05$ was considered statistically significant.

This work was approved by the Quality Improvement Committee of UPMC Health System.

\section{RESULTS}

Caspofungin was the first antimicrobial agent targeted by the ASP. This choice was driven in part by a marked increase in its use after a decision in 2008 by the hospital Pharmacy and Therapeutics Committee to place liposomal amphotericin B on our restricted antimicrobial list with the intent of redirecting empirical antifungal use toward caspofungin (Supplemental

Table 1, Fig 1 A and B). The mean caspofungin start rate for the entire pre-ASP period (including data before and after caspofungin's designation as recommended empirical antifungal agent) was 8.0 patients per 1000 patients compared with 18.7 in the post-ASP period $(P$ $<.001$ ), and the mean drug use rate was 14.0 and 29.3 patient-days per 1000 patient-days in the pre and post-ASP periods, respectively $(P=$ .003). After backward elimination of the "level change" variable (ie, the jump at time of ASP initiation), the parsimonious segmented regression model more clearly demonstrated a deceleration (ie, decreased slope) for drug starts and drug use after day 3 auditing was initiated $(P=.001$ and $P=.014$, respectively) (Fig $1 \mathrm{~A}$ and $B)$. In high-use physician services, increasing annual trends in usage were significantly blunted post-ASP (data not shown).

Vancomycin was the second targeted antimicrobial for day 3 auditing based on recognition of very high baseline use compared with other pediatric hospitals with highest institutional usage in critical care areas (unpublished data). Overall, the mean drug start rate was reduced from 137.7 patients per 1000 patients pre-ASP period to 121.4 in the post-ASP period $(P=.005)$, with mean drug use reduced from 138.2 patient-days per 1000 patient-days before intervention to 104.2 post-ASP $(P<.001)$. These changes represent a relative reduction of $12 \%$ for vancomycin drug starts and $25 \%$ for drug use. Parsimonious segmented regression analyses demonstrated constant drug start and drug use rates during the preintervention period compared with a significant downward trend for both measures after implementation of Day 3 auditing (Supplemental Table 2, Fig $2 \mathrm{~A}$ and B). Exploratory analyses indicated observed reductions in usage occurred across all patient subpopulations (ie, postintervention slope $<0$ ), with greatest decline observed in solid organ transplant recipients receiving critical care.

Meropenem was the third drug selected for day 3 auditing based on concerns that overuse of this agent could lead to development of resistance in an antibiotic of last resort. As meropenem had required preauthorization before implementation of the ASP, its overall use was modest both before and after implementation of day 3 auditing. Despite this, the mean drug start rate dropped from 14.3 to 11.3 patients per 1000 patients for the pre-ASP and post-ASP periods, respectively $(P=.067)$, and the mean drug use rate fell from 20.0 to 13.8 patient-days per 1000 patientdays for the pre- and post-ASP periods, respectively $(P=.021)$, corresponding to a relative reduction of $21 \%$ for meropenem drug starts and $31 \%$ for drug use. The drop in association with initiation of day 3 auditing was confirmed by segmented regression modeling although no changes were observed 
A

Caspofungin Drug Starts

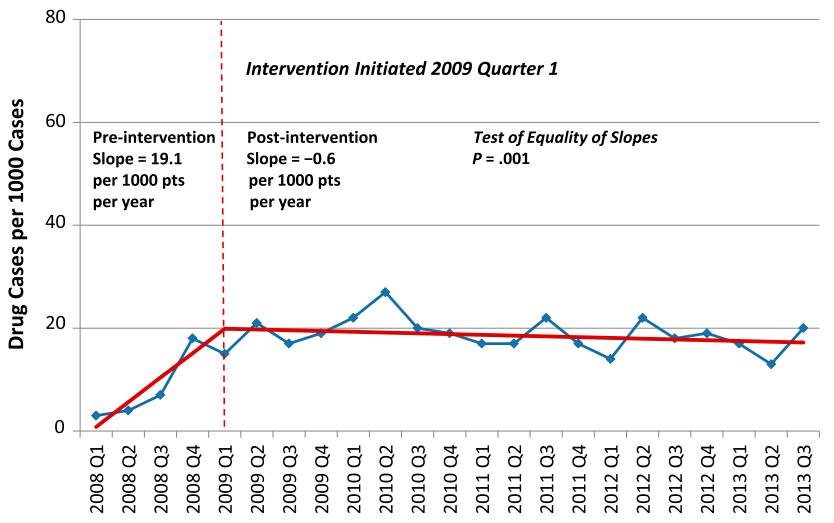

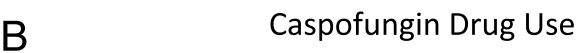

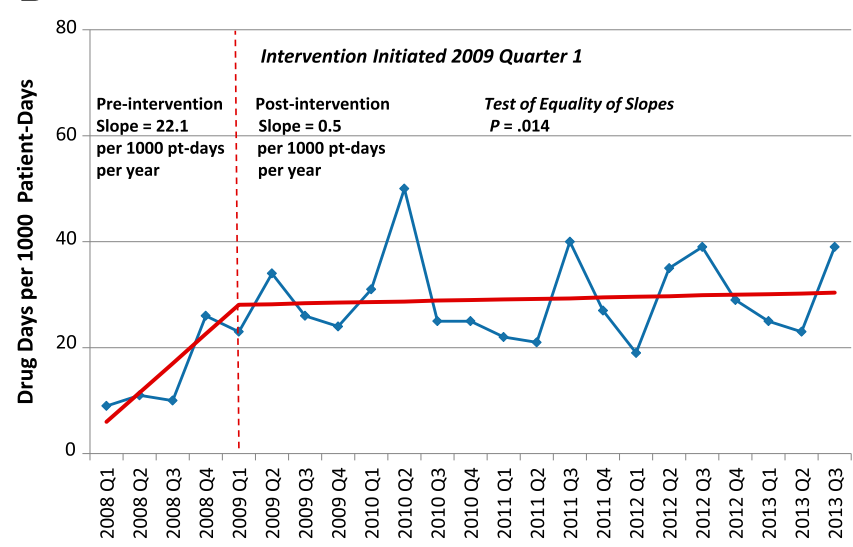

FIGURE 1 A AND B,

Caspofungin prescription data were collected from 2008 Quarter 1 through 2013 Quarter 3, and the Caspofungin ASP intervention was initiated in 2009 Quarter 1 (red dashed line). The y-axis for Fig 1A provides caspofungin cases per 1000 total cases, whereas for Fig 1B it provides caspofungin days per 1000 total patient-days. The $x$-axis provides the quarter/year of observation. The observed quarterly data (blue line with diamonds) and the fitted parsimonious segmented regression model (red solid line) for the drug start rates are shown in the top panel and the drug use rates are shown in the bottom panel.

A

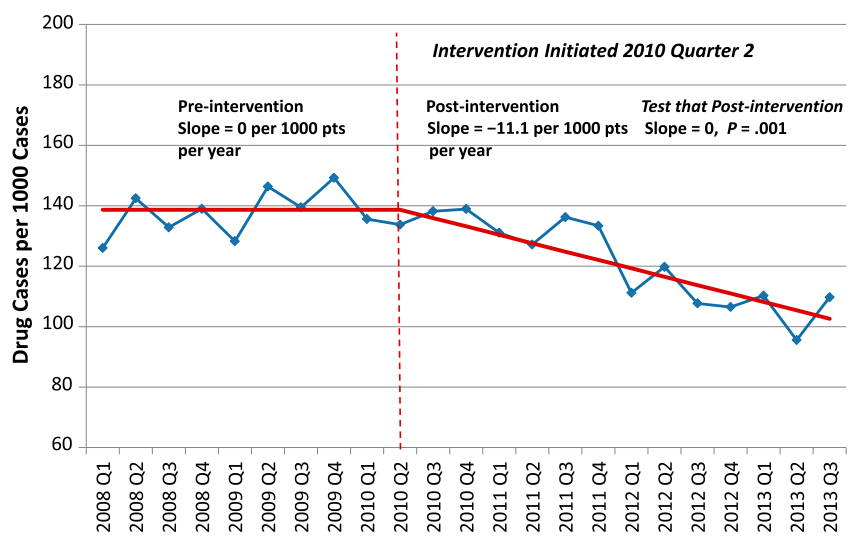

B

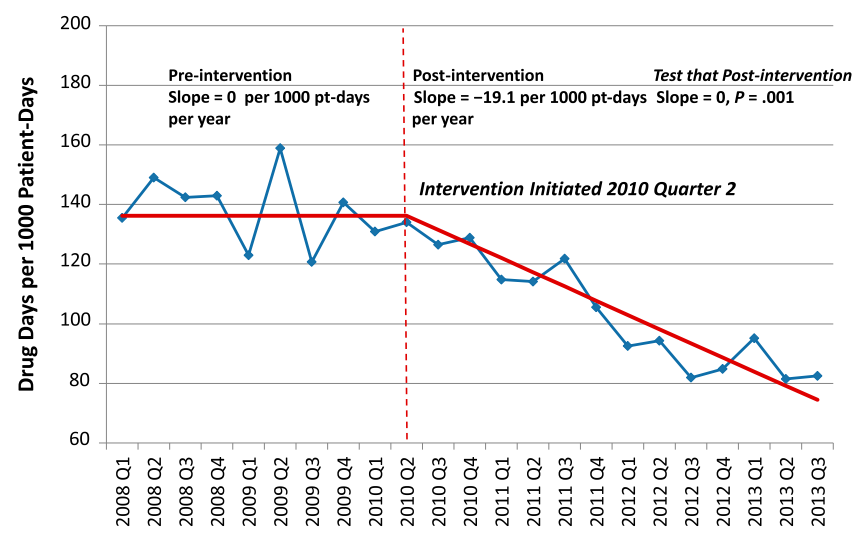

FIGURE 2 A AND B,

Vancomycin prescription data were collected from 2008 Quarter 1 through 2013 Quarter 3, and the Vancomycin ASP intervention was initiated in 2009 Quarter 2 (red dashed line). The y-axis for Fig 2A provides vancomycin cases per 1000 total cases, whereas for Fig $2 \mathrm{~B}$ it provides vancomycin days per 1000 total patient-days. The $x$-axis provides the quarter/year of observation. The observed quarterly data (blue line with diamonds) and the fitted parsimonious segmented regression model (red solid line) for the drug start rates are shown in the top panel and the drug use rate are shown in the bottom panel.

in drug start and use rates comparing the pre-ASP period and post-ASP time periods (Fig 3 A and B, Supplemental Table 3). Meropenem was most frequently prescribed in the PICU for abdominal transplant candidates and recipients; the reduction in meropenem use after the initiation of the ASP in this subpopulation was consistent with the findings in the overall population. Meropenem use in other patient populations was too limited to allow for meaningful subgroup analysis.
To assess whether potential changes in patient characteristics might explain observed changes in antimicrobial use over time, a comparison of the average number of patients per quarter and patient days per quarter before and after the introduction of our ASP was performed. The average number of total patients per quarter and total patient-days per quarter were similar before and after the intervention (3282 vs 3410 for total patients per quarter and 21502 vs 22412 for total patient-days per quarter, respectively). Among the higher-risk population, the average number patients per quarter and patientdays per quarter were greater after implementation of day 3 auditing for ICU (576 vs 638 for total patients per quarter and 3512 vs 3828 for total patient-days per quarter, respectively), NICU (168 vs 205 for total patients per quarter and 1933 vs 3201 for total patient-days per quarter, respectively), and oncology/ bone marrow transplantation 
A

Meropenem Drug Starts

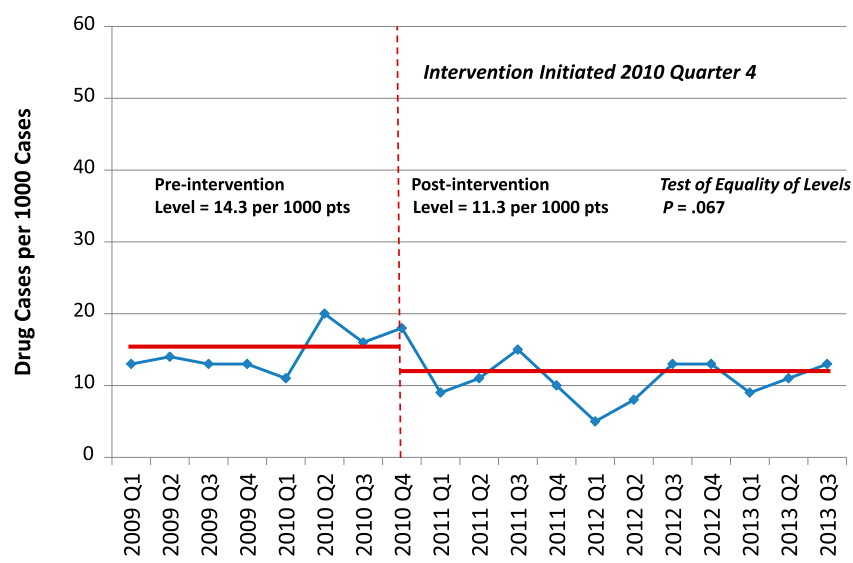

B

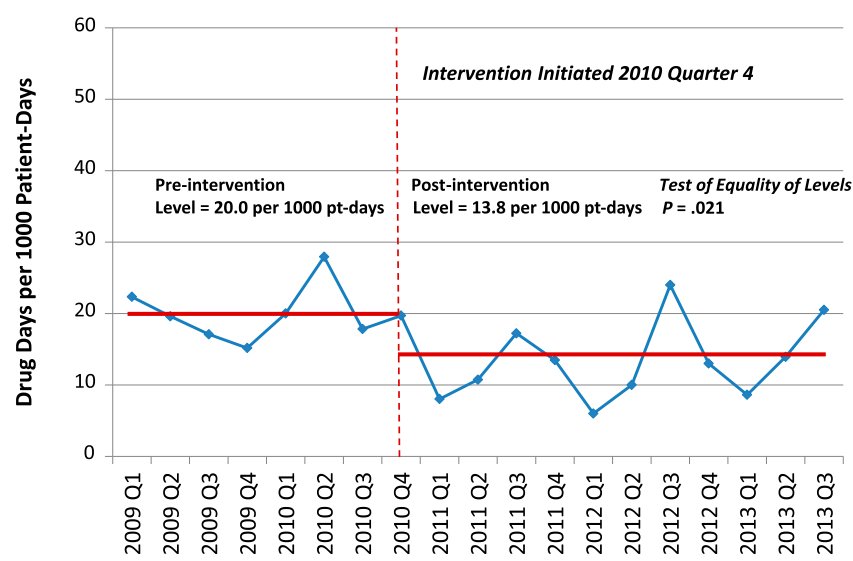

FIGURE 3 A AND B,

Meropenem prescription data were collected from 2009 Quarter 1 through 2013 Quarter 3, and the Meropenem ASP intervention was initiated in 2010 Quarter 4 (red dashed line). The y-axis for Fig 3 A provides meropenem cases per 1000 total cases, whereas for Fig 3B it provides meropenem days per 1000 total patient-days. The $x$-axis provides the quarter/year of observation. The observed quarterly data (blue line with diamonds) and the fitted parsimonious segmented regression model (red solid line) for the drug start rates are shown in the top panel and the drug use rates are shown in the bottom panel.

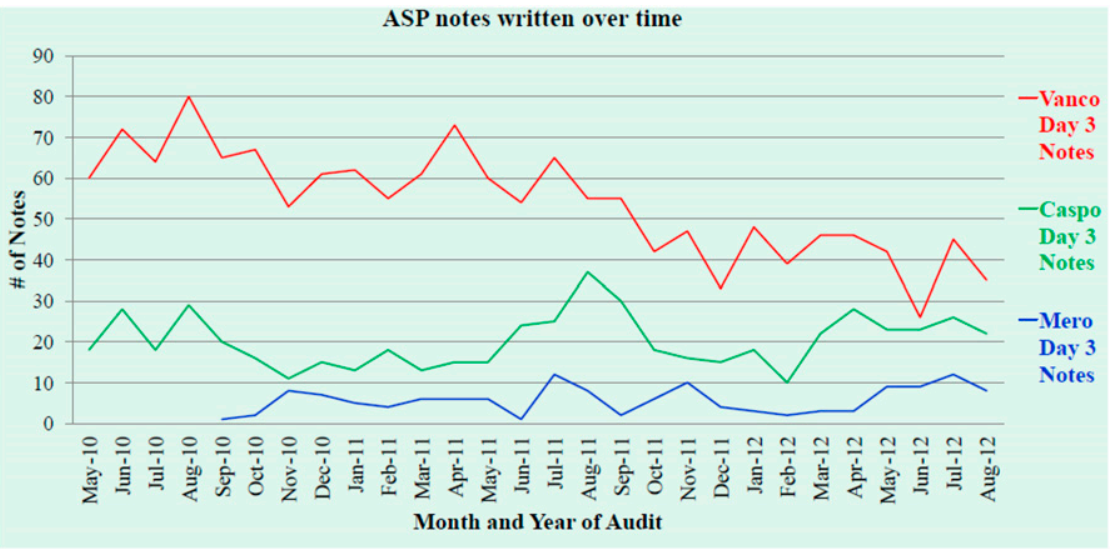

FIGURE 4

The number of ASP notes written over time.

patients were decreased in our abdominal transplant population (112 vs 94 for total patients per quarter and 1258 vs 957 for total patient-days per quarter, respectively).

Results of the audit of compliance with ASP recommendations demonstrated an overall acceptance rate of $90 \%, 93 \%$, and $100 \%$ for vancomycin, caspofungin, and meropenem, respectively. The decline in numbers of patients initially receiving vancomycin and overall drug use is more impressive when considering that these results were accompanied by a

\section{DISCUSSION}

progressive decrease in the number of vancomycin ASP notes over time (Fig 4).

Initiation of our alternative model ASP was associated with a decline in drug starts and overall drug use for the 3 targeted antimicrobial agents evaluated in this study. Our analyses demonstrated blunting of a preexisting increasing trend for caspofungin drug starts, as well as use, and a significant downward trend for vancomycin drug starts (relative change $-12 \%$ ) and use
$(-25 \%)$, with the largest reduction in critical care areas. Although meropenem use was already low due to preexisting requirements for preauthorization, a decline in drug use $(-31 \%, P=.021)$ and a nonsignificant decline in drug starts $(-21 \%, P=.067)$ were noted. Our analysis further demonstrated levels of acceptance of ASP recommendations of $90 \%, 93 \%$, and $100 \%$ for vancomycin, caspofungin, and meropenem, respectively. Taken together, the impact on antimicrobial starts and overall use along with the high level of acceptance of recommendations strongly support the effectiveness of this alternative model of ASP.

The use of ASPs is an increasingly important strategy to optimize use of antimicrobial agents. A recent survey of free-standing children's hospitals revealed that $38 \%$ had formal ASP, whereas an additional $36 \%$ were actively implementing programs. ${ }^{13}$ Common antimicrobial agents monitored included vancomycin, linezolid, daptomycin, fluoroquinolones, and carbapenems. Prospective audit and feedback, formulary restriction, and clinical guidelines were commonly cited strategies (87\%) $\cdot{ }^{13}$ In 2008, an ASP 
was constructed at this pediatric hospital by using key strategies recommended by the $\operatorname{IDSA}^{1}$ (eg, education, drug restriction, and auditing). However, the institution chose a nontraditional model to operationalize the program, integrating ASP activities into the daily function of service-based clinical pharmacists and of ID physicians during weekends and holidays. Multiple ASP strategies, including development of consensus guidelines for drug use with key physician stakeholders, requirement for approval before initiation of drug use for targeted antimicrobial agents, and ASP auditing on day 3, as well as ongoing education were used.1,11 The hospital's EMR and data warehouse were leveraged to identify patients requiring day 3 auditing, document ASP recommendations, and track and analyze trends in drug use over time.

An important primary endpoint for assessing the success of an ASP, regardless of model, is its impact on antimicrobial use. Although our ASP has worked to educate prescribing physicians about the use of antimicrobial agents in general, our outcome analyses focused on the 3 agents selected for day 3 auditing. The primary variables analyzed were drug starts and use (overall and for individual clinical services) for each targeted antimicrobial agent. Although our analyses suggest that implementation of day 3 auditing had a positive significant effect on all 3 agents, the impact on vancomycin was most dramatic. A stable but very high rate of both vancomycin starts and use were noted for the 2 years before implementation of day 3 auditing. After the intervention, a significant and ongoing steep drop in both the number of patients started on vancomycin and its overall use has been documented for the whole hospital and for most of our highrisk clinical services. The observed reductions in vancomycin starts and use are similar to those reported by
Di Pentima and Chan, ${ }^{3}$ describing a 33\% decline in vancomycin use as measured in doses administered per 1000 patient-days per year in a pediatric hospital after 3 years of auditing by using a "traditional" model of ASP. By comparison, we demonstrated an $\sim 25 \%$ decline in the use of vancomycin as measured by drug days per 1000 patient-days in a similar time period. Additionally, we have anecdotally observed that our physician services are more readily discontinuing vancomycin therapy before day 3 audits, suggesting that physicians are deescalating therapy when appropriate as part of daily patient care, independent of a formal ASP eNote.

The incorporation of daily ASP activities into the work of the service-based clinical pharmacists who participate in daily rounds takes advantage of established collaborative relationships between pharmacists and physician services, connecting ASP activities with pharmacist responsibility allowing for more informed prospective patient care reviews and assessment. We believe that use of service-based pharmacists for execution of our ASP allows for a more effective integration of antimicrobial stewardship into clinical decisionmaking and may be a major reason for observed high rates of compliance with ASP recommendations and reduction in use of targeted antimicrobial agents. As many children's hospitals also include service-based pharmacists as part of clinical care teams, the model of ASP implemented at our institution may be an effective alternative approach for these facilities.

Potential negative issues could be associated with the implementation of this alternative ASP compared with the traditional model that uses 1 dedicated physician and pharmacist. The current model requires that all service-based pharmacists and ID physicians be educated and trained in the conduct of the ASP, which might be challenging if turnover were frequent or if large numbers of individuals need to be reeducated due to infrequent service obligation. Another concern was possible hesitation by service-based pharmacists to make recommendations that diverge from the therapeutic plan of providers worked with on a daily basis. This concern was not borne out, as noted by the decline in use of targeted antimicrobial agents highlighted in this article. Finally, the lack of additional FTE support could limit the scope of ASP efforts compared with programs using dedicated personnel.

In contrast to the current ASP model, use of dedicated ASP pharmacists is common in both adult and pediatric stewardship programs. Newland et $\mathrm{al}^{13}$ reported that 13 of 16 freestanding pediatric hospitals with ASPs had dedicated pharmacist FTEs (median 0.5, range 0.1-1.5 FTE). The described model provides an effective alternative that may be particularly attractive when resources to support additional clinical pharmacists and physicians are limited. Other institutions also have adopted models unique to their culture and resources with success. DiazGranados and $\mathrm{Abd}^{14}$ reported a similar model of multiple clinical pharmacists' participation in ASP activities achieving significant costsavings. Bartlett and Siola ${ }^{15}$ also reported successful cost-containment initiatives with a $74 \%$ acceptance of ASP recommendations when multiple clinical pharmacists without specialized ID training participated. Their model differs from our model in that one payroll neutral ASP position was created with coverage of their ASP rotating among all dispensing and clinical pharmacists on a weekly basis.

This program leveraged the capabilities of the EMR and associated data warehouse for 
identification of patients requiring day 3 auditing and for documentation and communication of ASP recommendations to clinical teams. In addition, use of a data warehouse empowers the ASP program through the ability to generate real-time data regarding antimicrobial use through quarterly reports of patient numbers, usage, and duration of treatment of both hospital-wide and servicespecific populations of interest. The availability of these reports allowed for the equivalent of ongoing PDSA (Plan, Do, Study, Act) cycles ${ }^{16}$ to review our experience and further enhance the impact of the ASP.

The current study suffers from several potential limitations. Because the EMR and data warehouse were used as the basis for analyses of the program's effectiveness, we were not able to provide diseasespecific data on the impact of our intervention or correlate the impact of the ASP on length of stay, mortality, and so forth. However, current recommendations by the IDSA support assessment of whether ASPs result in desired changes in antimicrobial use. We believe that measurement of drug starts and usage accomplish this goal. Similarly, we do not have complete details of patient clinical characteristics before and after implementation of our ASP. Accordingly, some of the observed changes in antimicrobial use could be due to differences in patient characteristics in these 2 time periods. However, the analyses of total patients per quarter and patient-days per quarter and in particular the comparison of these data for higher-risk populations (eg, patients in the ICU, children with cancer) suggests more children in the postintervention period belonged to higher-risk groups suggesting that observed changes would not be due to a shift to lower-acuity patients who would require less antimicrobial therapy. An additional limitation to this work was the ability to look at quarterly drug prescription information only from 2008 onward, which prevented us from meeting the typical expectation of 12 observations before and after an intervention. Nonetheless, the magnitude of the observed changes after the intervention (especially for vancomycin) support the validity of the current analyses.

\section{CONGLUSIONS}

The implementation of a unique collaborative ASP model by using expertise of service-based clinical pharmacists and ID physician members successfully reduces drug usage of targeted drugs and provides an alternative to the "traditional" ASP model as originally envisioned. This approach to antimicrobial stewardship represents a viable alternative to current recommendations for the implementation of stewardship programs.

\section{ACKNOWLEDGMENTS}

This work was presented in part at the 48th and 49th Annual IDSA Meetings. We wish to acknowledge the critical contributions of our deceased dear colleague and co-author Dr James E. Levin.

\section{ABBREVIATIONS}

ASP: antimicrobial stewardship program

EMR: electronic medical record

FTE: full-time equivalent

ID: infectious disease

IDSA: Infectious Diseases Society of America

UPMC: University of Pittsburgh Medical Center

the final manuscript as submitted; Dr Brooks provided technical advice and carried out the statistical analyses described in this manuscript, provided the statistical methodologies included in the manuscript, reviewed and edited the content of the paper to confirm its accuracy relevant to the results of the analyses and provided content for the discussion, provided serial comments and recommendations and actively reviewed and edited the manuscript from the point of seeing the initial draft until its completion, and approved the final manuscript as submitted; Drs Darville, Thompson, and Michaels helped to conceptualize and implement the ASP and to analyze the data and assess the program on an ongoing basis, provided input toward the goal of the initial study and provided serial comments and recommendations and actively reviewed and edited the manuscript from the point of seeing the initial draft until its completion, and approved the final manuscript as submitted; Dr Levin helped to conceptualize and implement the ASP, developed both the electronic medical record used to implement the ASP and the software used to assess the impact of the program by using the hospital's data warehouse, before his untimely death, Dr Levin was actively involved in the regular review and assessment of the data and participated in the development of the statistical methodologies used in this manuscript; unfortunately, Dr Levin died before completion of this manuscript but the authors respectfully request including him as an author given his critical role in the development and assessment of this effort; and Dr Green was centrally involved in the conceptualization and implementation of both the ASP and this study, coordinated the development of the manuscript and worked with all of the co-authors to incorporate their suggestions and address editorial concerns, worked closely with Dr Brooks to implement the statistical analyses, provided direct input into each draft of the manuscript, and approves the final manuscript as submitted.

DOI: $10.1542 /$ peds.2015-0316

Accepted for publication Dec 21, 2015

Address correspondence to Michael Green, MD, MPH, Division of Infectious Diseases, Children's Hospital of Pittsburgh of UPMC, 4403 Penn Ave, Pittsburgh, PA 15224. E-mail: Michael.green@chp.edu

PEDIATRICS (ISSN Numbers: Print, 0031-4005; Online, 1098-4275). 
FINANCIAL DISCLOSURE: The authors have indicated they have no financial relationships relevant to this article to disclose.

FUNDING: No external funding.

POTENTIAL CONFLICT OF INTEREST: The authors have indicated they have no potential conflicts of interest to disclose.

\section{REFERENCES}

1. Dellit TH, Owens RC, McGowan JE Jr, et al; Infectious Diseases Society of America; Society for Healthcare Epidemiology of America. Infectious Diseases Society of America and the Society for Healthcare Epidemiology of America guidelines for developing an institutional program to enhance antimicrobial stewardship. Clin Infect Dis. 2007;44(2):159-177

2. File TM Jr, Srinivasan A, Bartlett JG. Antimicrobial stewardship: importance for patient and public health. Clin Infect Dis. 2014;59(suppl 3):S93-S96

3. Di Pentima MC, Chan S. Impact of antimicrobial stewardship program on vancomycin use in a pediatric teaching hospital. Pediatr Infect Dis J. 2010;29(8):707-711

4. Di Pentima MC, Chan S, Hossain J. Benefits of a pediatric antimicrobial stewardship program at a children's hospital. Pediatrics. 2011;128(6):1062-1070

5. Metjian TA, Prasad PA, Kogon A, Coffin SE, Zaoutis TE. Evaluation of an antimicrobial stewardship program at a pediatric teaching hospital. Pediatr Infect Dis J. 2008;27 (2):106-111
6. Hersh AL, De Lurgio SA, Thurm C, et al. Antimicrobial stewardship programs in freestanding children's hospitals. Pediatrics. 2015;135(1):33-39

7. Newland JG, Banerjee R, Gerber JS, Hersh AL, Steinke L, Weissman SJ. Antimicrobial stewardship in pediatric care: strategies and future directions. Pharmacotherapy. 2012;32(8):735-743

8. Newland JG, Hersh AL. Purpose and design of antimicrobial stewardship programs in pediatrics. Pediatr Infect Dis J. 2010;29(9):862-863

9. Newland JG, Banerjee R, Gerber JS, Hersh AL, Steinke L, Weissman SJ. Antimicrobial stewardship in pediatric care: strategies and future directions. Pharmacotherapy. 2012;32 (8):735-743

10. Centers for Disease Control and Prevention. Core elements of hospital antibiotic stewardship programs Available at www.cdc.gov/getsmart/ healthcare/implementation/coreelements.html. Accessed December 5, 2014

11. Wagner AK, Soumerai SB, Zhang F, Ross-Degnan D. Segmented regression analysis of interrupted time series studies in medication use research. $J$ Clin Pharm Ther. 2002;27 (4):299-309
12. Shardell M, Harris AD, El-Kamary SS, Furuno JP, Miller RR, Perencevich EN. Statistical analysis and application of quasi experiments to antimicrobial resistance intervention studies. Clin Infect Dis. 2007;45(7):901-907

13. Newland JG, Gerber JS, Weissman SJ, et al. Prevalence and characteristics of antimicrobial stewardship programs at freestanding children's hospitals in the United States. Infect Control Hosp Epidemiol. 2014;35(3):265-271

14. DiazGranados CA, Abd TT. Participation of clinical pharmacists without specialized infectious diseases training in antimicrobial stewardship. Am J Health Syst Pharm. 2011;68(18):1691-1692

15. Bartlett JM, Siola PL. Implementation and first-year results of an antimicrobial stewardship program at a community hospital. Am J Health Syst Pharm. 2014;71(11):943-949

16. Taylor MJ, McNicholas C, Nicolay C, Darzi A, Bell D, Reed JE. Systematic review of the application of the plando-study-act method to improve quality in healthcare. BMJ Qual Saf. 2014;23(4):290-298 


\section{A Quality Assessment of a Collaborative Model of a Pediatric Antimicrobial Stewardship Program}

Phuong-Tan Nguyen-Ha, Denise Howrie, Kelli Crowley, Carol G. Vetterly, William McGhee, Donald Berry, Elizabeth Ferguson, Emily Polischuk, Maria Mori Brooks, Jeffrey Goff, Terri Stillwell, Toni Darville, Ann E. Thompson, James E. Levin, Marian G. Michaels and Michael Green

Pediatrics 2016;137;

DOI: 10.1542/peds.2015-0316 originally published online April 6, 2016;

\begin{tabular}{|c|c|}
\hline $\begin{array}{l}\text { Updated Information \& } \\
\text { Services }\end{array}$ & $\begin{array}{l}\text { including high resolution figures, can be found at: } \\
\text { http://pediatrics.aappublications.org/content/137/5/e20150316 }\end{array}$ \\
\hline References & $\begin{array}{l}\text { This article cites } 15 \text { articles, } 5 \text { of which you can access for free at: } \\
\text { http://pediatrics.aappublications.org/content/137/5/e20150316\#BIBL }\end{array}$ \\
\hline Subspecialty Collections & $\begin{array}{l}\text { This article, along with others on similar topics, appears in the } \\
\text { following collection(s): } \\
\text { Administration/Practice Management } \\
\text { http://www.aappublications.org/cgi/collection/administration:practice } \\
\text { management_sub } \\
\text { Quality Improvement } \\
\text { http://www.aappublications.org/cgi/collection/quality_improvement_ } \\
\text { sub } \\
\text { Infectious Disease } \\
\text { http://www.aappublications.org/cgi/collection/infectious_diseases_su } \\
\text { b }\end{array}$ \\
\hline Permissions \& Licensing & $\begin{array}{l}\text { Information about reproducing this article in parts (figures, tables) or } \\
\text { in its entirety can be found online at: } \\
\text { http://www.aappublications.org/site/misc/Permissions.xhtml }\end{array}$ \\
\hline Reprints & $\begin{array}{l}\text { Information about ordering reprints can be found online: } \\
\text { http://www.aappublications.org/site/misc/reprints.xhtml }\end{array}$ \\
\hline
\end{tabular}




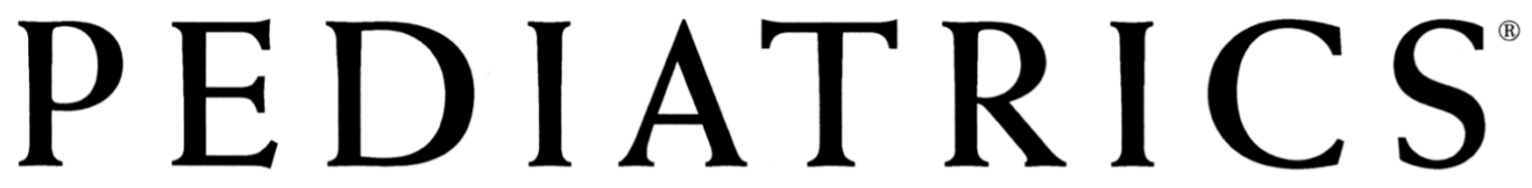

OFFICIAL JOURNAL OF THE AMERICAN ACADEMY OF PEDIATRICS

\section{A Quality Assessment of a Collaborative Model of a Pediatric Antimicrobial Stewardship Program}

Phuong-Tan Nguyen-Ha, Denise Howrie, Kelli Crowley, Carol G. Vetterly, William McGhee, Donald Berry, Elizabeth Ferguson, Emily Polischuk, Maria Mori Brooks, Jeffrey Goff, Terri Stillwell, Toni Darville, Ann E. Thompson, James E. Levin, Marian G. Michaels and Michael Green

Pediatrics 2016;137;

DOI: 10.1542/peds.2015-0316 originally published online April 6, 2016;

The online version of this article, along with updated information and services, is located on the World Wide Web at: http://pediatrics.aappublications.org/content/137/5/e20150316

Data Supplement at:

http://pediatrics.aappublications.org/content/suppl/2016/04/20/peds.2015-0316.DCSupplemental

Pediatrics is the official journal of the American Academy of Pediatrics. A monthly publication, it has been published continuously since 1948. Pediatrics is owned, published, and trademarked by the American Academy of Pediatrics, 141 Northwest Point Boulevard, Elk Grove Village, Illinois, 60007. Copyright $\odot 2016$ by the American Academy of Pediatrics. All rights reserved. Print ISSN: 1073-0397. 